Hospital Roberto del Rio.

Sección de Cirugía Infantil y Ortopedia.

Prof. Dr. A. Johow.

\title{
NUESTRA EXPERIENCIA SOBRE EL TRATAMIENTO DE LA HERNIA UMBILICAL EN EL NINO
}

\author{
Por el Dr. GUILLERMO ZIEGLER S.
}

Para discutir los diferentes métodos de tratamiento de la hernia umbilical, hay que exponer previamente la etiopatogenia de dicha hernia.

El Dr. Poblete. del Servicio de Puericultura de la Maternidad del Hospital Bartos Luco de Santiago, ha podjdo observar que los tecién nacidcs, que presentan an cordón unbilical con base saliente crónica de piel y aquellos otros que presentan granulomas o infecciones del muñón umbilical, quedan en un gran porcentaje con hernia umbilical.

La hernia umbilical es, pues, una deformación congénita de la pared abdominal, oue se puede acompañar de otros defectos del desarrollo. De aquí que de 132 enfermos hospitalizados en el Hospital Roberto del Rio por hernia umbilical desde el año 1932, un $10.6 \%$ presentaban, además. hernias inguinales: un $3,8 \%$, prolapsos rectales, $y$ un $1,5 \%$, bipospadias.

Estos enfermos, que presentan un desarrolio defectuoso de su pared abdorninal. están predispuestos congénitamente a presentar una hernia umbilical en cualquier momento de su vida, por un aumento de la presión intraabdominal lesfuerzo, embarazo, ascitis. obstrucción intestinal, ete.).

Por otra parte, la hernia umbilical del lactante puede desaparecer espontáneamente por el crecimiento y desarrollo 
de la aponeurosis abdominal, que tiende a cerrar el anillo herniario.

La hernia presenta generaimente el tamaño de un gatbanzo; puede alcanzar el tamaño de un huevo de gallina, mientras que en otras ocasiones es tan pequeña, que es de difícil diagnóstico, por asemejarse a un pliegue de la cicatriz umbilical. En el niño mayor, generalmente encontramos epiplón, el cual molesta durante el acto operatorio y en las hernias de los lactantes y en las de anillo amplio del niño mayor, es generalmente el intestino el que se hernia. pues en el lactante el epiplón mayor aun no se ha desarrollado.

La hernia umbilical produce molestias. No estamos de acuerdo con Glanzmann (5), el cual sostiene que es muy frecuente invocar a la hernia umbilical como causa de dolores de vientre y que raras veces constituyen la verdadera causa de dichas molestias". Nosotros podemos sostener actual. mente, con una gran probabilidad, que tal o cual molestia corresponde a una apendicitis y con la misma probabilidad podemos sostener que tal o cual molestia es producida por una hernia umbilical.

Son padecimientos generalmente poco molestos y fuga ces. raras veces graves, que se producen por atascamiento del epiplón o intestino, con las consiguientes tracciones sobre el meso sensible o por la pared delgada del ombligo, formada únicamente por piel y peritoneo, lo que expone a enfriamientos de las visceras herniadas.

Hemos revisado 132 historias de bernia umbilical, del Hospital Roberto del Río y hemos encontrado anotados en 76 historias las siguientes molestias:

39 casos con dolores vagos $e$ insidiosos.

17 casos con dolores al aumentar de volumen la hernia.

6 casos con dolor con la tos y el esfuerzo.

6 casos con dolor y vómitos, especialmente después de las comidas.

4 casos con crisis de dolor y diarrea con el frío.

2 casos con dolor al comer e inapetencia.

1 caso con dolor, que obligaba a guardar cama.

1 caso que se ponía pálida de dolor.

Estas mismas molestias las presentaban las enfermas adultas del Hospital Barros Luco y se encuentran descritas en las historias clinicas revisadas del mismo Hospital. 
Todas estas consideraciones previas son necesarias, pues en ellas se basa el tratamiento de la hernia umbilical dal niño. Debemos insistir y repetir de que se trata de un defecto congénito de la aponeurosis abdominal, susceptible de corregirse por el crecimiento en los tres primeros años de vida del niño.

De aquí sacamos cuatro conclusiones importantes, confirmadas por la práctíca:

1. El porcentaje de lactantes con hernia umbilical disminuye rápidamente con la edad, por curación espontánea.

2. E1 porcentaje de hernias umbilicales puede disminuir aún todavía más en número y tamaño. por medio del uso correcto y cuidadoso de un ombliguero en los recién nacidos o por medio del uso de una tela adhesiva, faja a lo huaso o braguero en los lactantes.

3. En todo niño menor de 3 años, que presente hernia umbilical, debe ensayarse primero el tratamiento ortopédico y únicamente fracasado éste se debe pensar en el tratamiento quirúrgico.

4. Terminado el desarrollo de la pared abdominal entre $\operatorname{los} 3$ y 4 años, hay que intervenir quirúrgicamente, interviniendo sobre un defecto de la pared abdominal, que ya no puede corregirse por medio del crecimiento.

Hechas estas consideraciones, pasaremos a hablar sobre el tratamiento de la hernia.

La mayoria de las hernias umbilicales pequeñas no producen molestias, se mantienen reducidas $y$ no aumentan de volumen con el llanto o por los esfuerzos. Esta clase de hernia tiande a la curación espontánea en los lactantes, por lo cual se deja sin tratamiento, sin ninguna faja, para no impedir el desarrollo de la musculatura abdominal. Es innecesario e indebido limitar la actividad de estos niños, ya que el mejoramiento de la musculatura general que motivan los ejercicios físicos es un factor importante en la curación.

Si la hernia del lactante es mayor y difícil de mantenerse reducida, producirá molestias. La evisceración mantizne abierta la brecha aponeurótica, impidiendo el cierre de! anillo por medio del crecimiento.

Estas hernias deben mantenerse reducidas: se plegará ia piel de la región umbilical, de modo que forme un surco longitudinal, en el que quiede escondido el ombligo $y$ se mantendrá en esta posición por medio de tiras de tela adhesiva de $5 \mathrm{cms}$. de ancho $\mathrm{y}$ de una longitud suficiente para 
que lleguen por ambos lados hasta las líneas axilares. En esta forma los tejidos sirven de cojín compresivo.

Los lactantes se pueden bañar con la tela, la cual se quitará rodas las veces que sea necesario para inspeccionar la piel $y$ aplicarla en sitios distintos, en caso de dermatitis. La tela queda generalmente aplicada 8 a 14 días. La consideramos contraindicada: 1. En caso de que el ombligo no esté completamente seco y sano. 2. En los recién nacidos y en los lactantes de piel delicada, en los cuales es de resultado a veces desastrcso. En estos casos usamos el ombliguero cortiente, fajas a lo huaso de varias vueltas o bragueros especiales.

Según Patterson y Gray 11937), santaron sin operación 105 enfermos de un total de 214 entermos controlados. es decir, más o menos un $50 \%$ (Morse). Esto coincide con nuestra observación de 18 casos bien controlados ya más de un año y de los cuales han curado ya 8 enfermos. Se trata de enfermos de 1 a 3 años de edad, que siguen en observación.

Contrariamente a lo que opinan muchos pediatras. las telas no impiden en absoluto los movimientos musculares. pues las telas adhesivas se fijan en la piel, que resbala encima de la capa músculo-aponeurótica: pues está separada de ella por el tejido celular y no impide los movimientos musculares.

Beadnell (1942) sostiene que una tela que no se fije al plano óseo vertebral, es decir, que no haga una vuelta completa, no mantiene reducida la hernia. Según nuestro parecer. esto no es exacto, pues la tela obra como cuerda elástica, reduciendo constantemente la hernia dentro del abdomen. La vuelta entera de Beadnell presenta ciertos peligros, pues no cede al meteorismo abdominal y causa generalmente más molestias que la hernia misma.

El botón o la moneda sujeta a una tela u ombliguero, ya no la usamos, pues éstos penetran en el orificio herniario, manteniéndolo abierto.

La gran mayoría de nuestros enfermos han sido tratados en la forma más arriba descrita. La indicación operatoria no pasa más allá de un $0.75 \%$, juntando todas las hernias umbilicales vistas en la policlínica de Cirugía del Hospital Roberto del Río. He aquíla estadistica de los enfermos cperados en los últimos 10 años en el Hospital Roberto del Río. 
Año

Antes de los

6 mises 1 año $2 \quad 3-4 \quad 5-6 \quad 7-8 \quad 9-12$ Total

1935

1936

1937

1938

1939

1940

1941

1942

1943

1944

Total

\begin{tabular}{|c|c|c|c|c|c|c|c|}
\hline- & - & - & - & 1 & 1 & 3 & 5 \\
\hline 2 & 1 & 1 & 1 & 1 & $\overline{2}$ & 2 & $\begin{array}{l}3 \\
8\end{array}$ \\
\hline & 2 & - & 4 & 2 & 2 & & 11 \\
\hline & & - & & 1 & 3 & & 8 \\
\hline & 2 & $\overline{2}$ & _- & $\overline{-}$ & 二 & $\frac{1}{3}$ & \\
\hline & $\bar{\gamma}$ & $\sqrt{2}$ & $\rightarrow$ & 1 & 1 & & $\therefore 8$ \\
\hline & 2 & 5 & 1 & 4 & 1 & 14 & 27 \\
\hline & 10 & a & 7 & 11 & 11 & & \\
\hline
\end{tabular}
pues:

La cirugía no tiene un papel tan amplio en estos casos.

1. Muchos sanan espontáneamente o por medio de los métodos ya enunciados.

2. Las molestias son generaimente minimas, de aquí que no hay ninguna prisa de operar las hernias umbilicales de los lactantes. La hernia umbilical, tan frecuente en el niño, no se estrangula jamás. Revisamos las estadisticas del Hospital Roberto del Río desde 1932 y no hemos encontrado ninguna hernia umbilical estrangulada. Revisamos tambien las historias de los adultos operados en el Hospital Barros Luco. En dicho hospital, un $40 \%$ de las hernias umbilicales fueron cperadas por estar estranguladas, siendo generalmente el epipión el órgano estrangulado y muy raras veces el intestino. La enferma más joven tenía 31 años, lo que coincide con la observación de Corachan, que antes de $\operatorname{los} 45$ años la estrangulación es excepcional.

Nosotros operamos únicamente en los siguientes casos:

1. Casos en que la hernia umbilical altere el desarrollo normal y el crecimiento del lactante.

2. Lactantes con hernias que no ceden con el tratamiento ortopédico. Si después de cierto tiempo (más o menos 6 meses de tratamiento con tela). el anillo herniario no tiende a cerrar, no conviene esperar, pues los resultados operatorios a esta edad son siempre buenos, reproduciéndose la hernia excepcionalmente.

3. Lactantes con hernias voluminosas y molestas, en los cuales el tratamiento quirúrgico es más rápido y seguro. 
4. Niños mayores de 3 años. en los cuales el cierre del anilio herniario por medio del crecimiento es más difícil de obtener. Ombredanne considera la edad de 6 años como limite.

5. La hernia umbilical es más frecuente en la mujer (61\%. Hospital Roberto del Río). Somos más quirúrgicos en caso de tratarse de niñas que necesitan una pared abdominal fuerte, sin defectos para resistir en su vida posterior el gran aumento de la presión intrabdominal durante el embarazo y luego el gran esfuerzo del parto. Revisando las historias clínicas del Hospital Barros Luco, un $96 \%$ de las hernias umbilicales eran mujeres y solamente un $4 \%$ hombres. Las estrangulaciones herniarias fueroti únicamiente de mujeres. Los peligros de la hernia umbilical en el adulto son la estran. gulación del epiplón con inflamación de éste (epiploitis). Se producen adherencias entre el saco y el epliplón, que deja cavidades. en que se estrangulan los intestinos. La región presenta en los adultos eczema y ulceraciones de la piel por el sudor $y$ la inactividad de estos enfermos produce obesidad $y$ miocarditis.

En caso de estar contraindicada la operación por lesiones de la piel u otra causa, preferimos esperar, ya que la espera no encierra ningún peligro en el niño.

En cuanto a la técnica operatoria, usamos desde el año 1944 una técnica, que es combinación de varios métodos. La incisión de la piel la practicamos según Blodgett (1941), síguiendo el borde superior del ombligo. Esta incisión no deja defectos estéticos, da un magnífico campo y con un aseo prolijo preoperatorio de la piel del ombligo no tuvimos ninguna supuración. Además, es generalmente en la parte superior del ombligo, sitio en que penetran los vasos umbilicales, donde se encuentra el anillo herniario.

No extirpamos el ombligo, como lo hace Ombredanne. pues en el niño la hernia siempre es reductible.

El cierre de la aponeurosis la practicamos según la técnica usual de Mayo. suturando en sentido transversal, pues la gran mayoria de los anillos herniarios tienen forma elíptica de eje mayor transversal, observación propia no mencionada en ningún trabajo, y que se explica por el entrecruzamiento de las fibras musculares a nivel de la línea media. Fuera de esto tiene ventaja suturar la aponeurosis en sentido horizontal, pues las fuerzas musculates obran en la misma dirección. Usamos generalmente catgut y únicamente en aquellos casos en que tememos la reproducción, colocamos puntos 


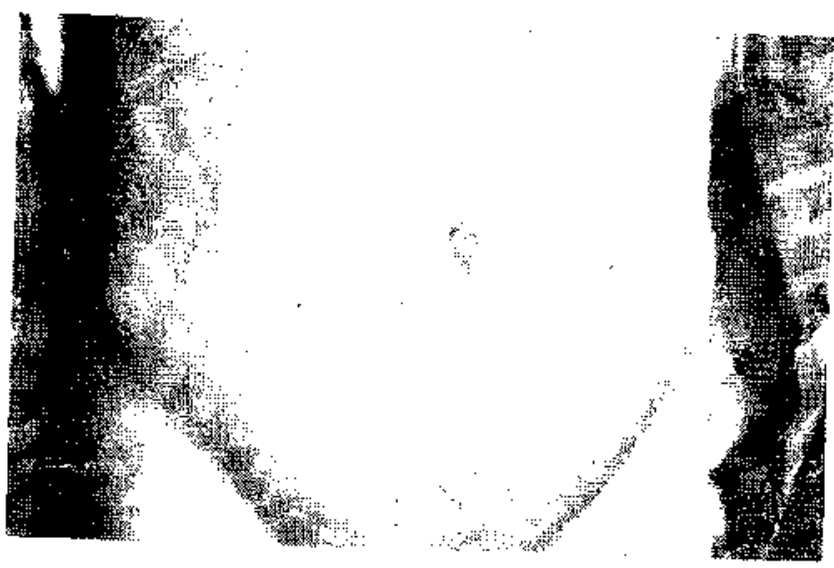

J. T. - 11.VIII.44

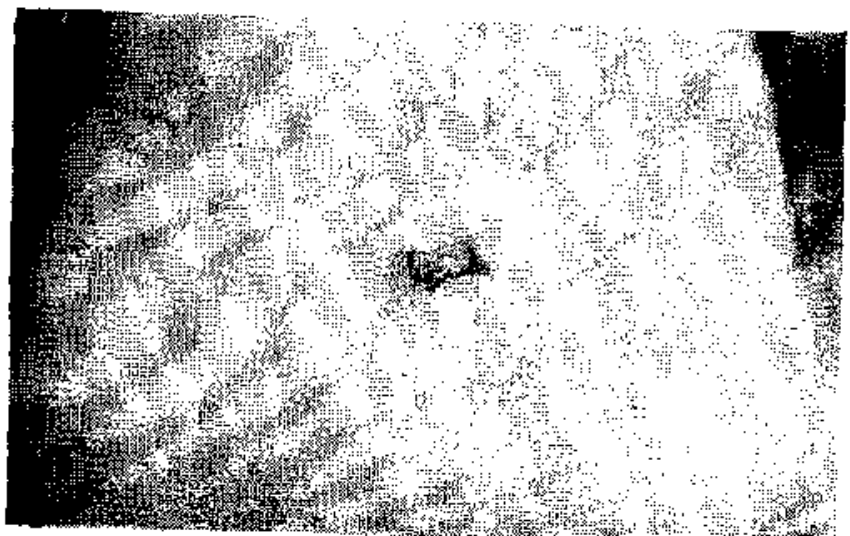

S. O. - iO-XI-4.4.

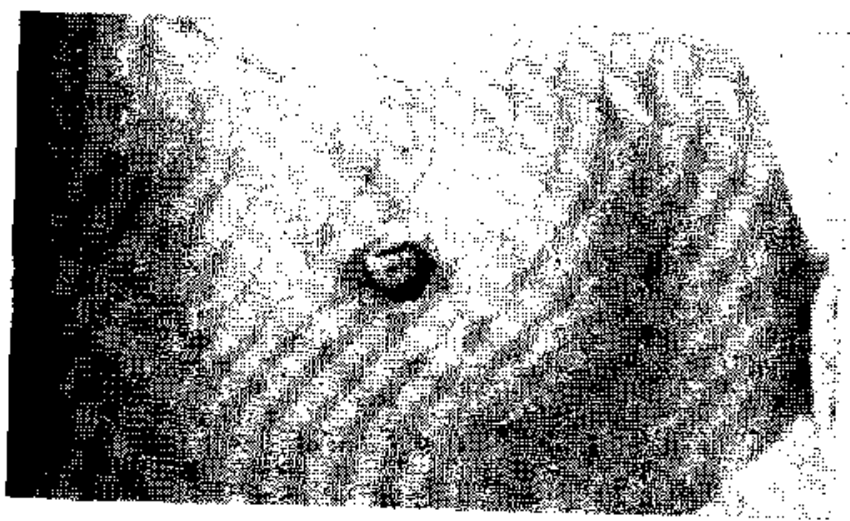

A. L. - $28 \cdot \mathrm{XI}-44$. 
de hilo a la aponeurosis. No suturamos los músculos rectos, como lo practica Blodgett en las pequeñas hernias del ombligo, pues, los músculos rectos generalmente se encuentran lejos de la linea media y al juntarlos dejan ombligos prominentes y deformes. Después de la operación conviene poner tela adhesiva ancha para comprimir cualquier espacio libre y evitar con esto la formación de un hematoma molesto.

Hemos operado 21 enfermos con este método, con la nueva incisión y hemos quedado completamente satisfechos. He aquí los números de las historias clinicas:

$44 / 2465$ J. T.
$44 / 2747$ M. U.
$44 / 2844$ I. J.
$44 / 3105$ N. M.
$44 / 3294$ L. O.
$44 / 3255$ N. H.
$44 / 3316$ A. S.
$44 / 3335$ R. F.
$44 / 3400$ P. A.
$44 / 3437$ C. S.
$44 / 3572$ F. C.

44/3514 J. D.
$44 / 2057$ L. B.
44/3937 M. P.
$44 / 4263$ L. A. D.
$45 / 342$ H. M.
$45 / 420$ Z. V.
$45 / 467$ M. G.
$45 / 853$ M. R. A.
$45 / 1266$ M. B. F.
$46 / 295$ E. P. S.

La ligadura subcutánea del antillo herniario (Fraser 1925), procedimiento ensayado en Chile por el Prof. Dr. Ernesto Prieto y presentado a la Sociedad de Pediatria en 1938. lo bemos abandonado definitivamente. Es un procedimiento antiquirúrgico, ciego, como demuestra una muerte por obstrucción intestinal (Obs. clínica 41/1419) por ligadura del intestino delgado. Fuera de esto se reprodujeron cuatro hernias, presentando posteriormente mayores molestias por adherencias del epiplón ligado.

Tampoco usamos el tratamiento por medio de inyeccio. nes esclerosantes descrito por Bennet-Jones (1944), método que presenta los mismos inconvenientes del método anterior. fuera de ser peligroso por la sustancia esclerosante misma. Está limitado a hernias de anillo pequeño, expone a un fracaso de un $30 \%$ y su mayor desventaja está en que el temperamento de los niños no está hecho para sufrit inyecciones repetidas (dos o cuatro), que se deben colocar con anestesia general. Preferimos en estos casos la intervención quirúrgica con una sola anestesia. 


\section{Resumen}

Se fijan normas para el correcto tratamiento de la herria umbilical en el niño. precisando las indicaciones operatorias. Se describe una técnica propia usando la incisión estética de Blodgett.

\section{Bibliografía}

1. BENNET JONES - BRTT. M. J. - London. 1944. Pág. 78

2. BLODGETT.-- Surg. Gynec, ఈ Obst. 1941. March.

3. BEADNELL - BRIT. M. J. 1941. Pág. 563.

4. FORGUE.- Manual de Fatología Externa. 1938. Edit. Espasa Calpé.

5. GLANZMANY.- Iecciones de Pediarria. 1942.

6. LADD \& GROSS.- Abdominal Surgery of Infancy and Childhood. Saunders, 1941.

7. MOVER - BRIT. M. J. 1943. Pág. 771.

8. OMBREDANNE.-- Tratado de Cirugia Infantil. 1932. Masson.

9. PRIETO, E. - Revista Chilena de Pediatría. 1938. Pág. 647 